\section{Cahiers de Narratologie}

Analyse et théorie narratives

$10.1 \mid 2001$

La voix narrative

\title{
La voix de l'enfant dans les romans de Soledad Puértolas
}

\section{Christine Di Benedetto}

\section{(2) OpenEdition \\ 1 Journals}

\section{Electronic version}

URL: http://journals.openedition.org/narratologie/6965

DOI: 10.4000/narratologie.6965

ISSN: 1765-307X

\section{Publisher}

LIRCES

\section{Printed version}

Date of publication: 1 January 2001

Number of pages: $365-376$

ISBN: 2914561032

ISSN: 0993-8516

\section{Electronic reference}

Christine Di Benedetto, "La voix de l'enfant dans les romans de Soledad Puértolas ", Cahiers de Narratologie [Online], 10.1 | 2001, Online since 31 October 2014, connection on 23 February 2021. URL: http://journals.openedition.org/narratologie/6965 ; DOI: https://doi.org/10.4000/narratologie.6965 


\title{
LA VOIX DE L'ENFANT DANS LES ROMANS DE SOLEDAD PUÉRTOLAS
}

\author{
Christine DI BENEDETTO \\ Université de Nice-Sophia Antipolis
}

Soledad Puértolas, une des romancières espagnoles contemporaines les plus appréciées, offre dans ses huit premiers romans des mondes qui se développent, au-delà de l'intrigue, grâce à des réseaux de liens entre les personnages. Elle confirme d'ailleurs elle-même que ce qui l'intéresse c'est que la force de sa création provienne d'eux ${ }^{1}$.

Dans une vision que nous voulons d'ensemble de son œuvre romanesque, il est une catégorie qui occupe une place à part, celle des personnages d'enfants. Nous entendrons par enfants tous ceux qui évoluent à l'âge de l'enfance ou de l'adolescence - en laissant de côté l'acception filiation, car elle, dure toute une vie. Une lecture rapide peut laisser croire que ces personnages d'enfants sont très minoritaires et silencieux. Or, si l'on étudie la voix narrative, on constate, avec un peu plus d'attention, qu'ils expriment toujours clairement quelque chose, qu'ils soient complètement absents, simplement évoqués ou vraiment présents dans la narration.

Nous allons chercher à déterminer la place réelle que l'enfant occupe dans ces romans pour chacune de ces éventualités, afin de comprendre ensuite comment il se fait entendre, quelle est sa voix - celle-ci n'étant pas uniquement parole - et à qui elle s'adresse. Nous procéderons pour cela à deux analyses simultanées : celle de la matière du récit et celle des mécanismes narratifs qui servent à raconter l'enfance et les souvenirs.

${ }^{1}$ Entrevista a Soledad Puértolas, «La mirada del otro », Leer, JuilAoût 1999, p. 104. 


\section{L'enfant absent : échec et frustration :}

Nuançons d'abord l'idée d'absence de personnages d'enfants. L'absence totale n'existe pas chez Soledad Puértolas. Même dans Si al atardecer llegara el mensajero, où le sujet apparaît le moins, Arturo Nizranín fait allusion à ses deux enfants qu'il a si mal connus faute d'avoir assumé ses responsabilités de père et qui, pour finir, lui ont échappé. Cette absence est échec, comme c'est le cas pour Pauline Duvivier, dans Burdeos. Célibataire à l'automne de sa vie, elle n'a pas construit de famille et sait qu'elle ne laissera rien derrière elle ${ }^{2}$.

Le second cas d'absence d'enfant dans le présent de la narration est en fait la simple évocation. Celle-ci est le plus souvent matérialisée dans la relation mère/enfants. Si la fratrie est nombreuse, elle est présentée comme une charge pour la mère, une négation de son identité de femme. C'est le cas pour Raquel, dans Queda la noche. Nous ignorons tout de ses cinq enfants. Par son entourage, on apprend qu'elle n'est jamais disponible car elle a toujours des problèmes avec l'un d'entre eux ; elle-même confie qu'elle se sent piégée, depuis qu'elle a cessé de travailler, dès sa première grossesse. A l'identique, Madeleine, femme de ménage dans Burdeos, se débat entre ses quatre garçons et un mari alcoolique. Et les exemples sont nombreux... Araceli dans Queda la noche puis Días del Arenal, au départ simple fille de village, lutte par tous les moyens pour sortir de sa condition et offrir un avenir meilleur à ses six enfants. Gracia, dans Días del Arenal encore, étouffe dans sa sage vie de mère de famille avec quatre enfants, ce qui la conduira à prendre un amant. Sa sœur Herminia est aussi entravée dans ses ambitions, dans son cas sa vocation de poète, par cinq enfants. Enfin, Marta Berg, dans le roman du même nom, semble aussi ne vivre que par ses quatre enfants.

Atypique dans cette série de familles nombreuses, nous trouvons Jacobo, le fils unique de Susana dans Días del Arenal. Cet enfant n'a en réalité pas de poids dans l'intrigue ;

2 Burdeos, Anagrama, Barcelona, 1986, p. 12. 
il permet seulement à Susana, divorcée, de se positionner en tant que mère. Cependant nous dirons que, bien qu'indirecte, sa seule existence est voix.

L'enfant, donc, même absent, dit qui est sa mère parce qu'il détermine la vie de celle-ci. En échange, dans cette relation double, il se positionnera aussi par rapport à elle, dans un système d'oppositions et d'attirances.

La voix de l'enfant est donc majoritairement dans ce cas situationnelle et non-verbale.

\section{L'enfant en situation : voix rapportée et maladie :}

A l'opposé des situations précédemment décrites, il y a l'enfant en tant que personnage agissant dans le présent de la narration, mais c'est exceptionnel. On ne trouve en effet qu'un seul exemple : celui des deux sœurs Mónica et Natalia dans La Señora Berg, filles du narrateur, Mario. Nous faisons leur connaissance quelques temps avant que leur mère, Claudia, ne quitte le foyer, quand elles ont six et huit ans respectivement et nous les suivons jusqu'à ce qu'elles soient devenues jeunes filles. Comment apparaissent-elles ? C'est un narrateur adulte, leur père, qui nous en parle. En effet, c'est lui qui assume leur vie quotidienne après le départ de sa femme et donc les voit évoluer. Mais elles-mêmes s'expriment-elles ? Deux fois seulement, et de façon assez limitée, Soledad Puértolas emploie le discours direct et leur laisse la parole :

«Me lo dijeron las niñas : « Mamá se ha casado, nos ha dicho que se ha casado. $» »^{3}$.

$\mathrm{Ce}$ ne sont que quelques mots; puis, une seconde fois, lorsque Mario les interroge car il soupçonne que Claudia a des problèmes avec son nouveau mari, on assiste à un bref échange à trois en onze tirades relativement courtes. (p. 229) Or, s'il y a peu de discours direct, ou rapporté, il serait erroné de croire que ce sont des personnages sans voix.

Après le départ du foyer de leur mère, devenues aux yeux des autres des enfants abandonnées, les filles se taisent et ne se plaignent pas. Leur voix est silence, peut-être pour protéger leur père, se dit celui-ci, ou bien parce que :

${ }^{3}$ Días del Arenal, Barcelona, Ed. Planeta, 1992, p. 182. 
« (...) à cet âge, en pleine enfance - $(\ldots)$ - il existe une capacité naturelle pour la dissimulation, l'enfance est le royaume des jeux et des rôles. »4.

Une autre voix, rapportée cette fois, surgit dans ce que le père dit de ses filles. La voix des personnages-enfants cède le pas à la voix du narrateur-adulte (pour employer la terminologie de Genette au sujet de la voix narrative). Narrateur non omniscient, celui-ci garde une grande prudence quant à ce qu'il perçoit ; il multiplie les formules de recul (probablement, peut-être...), les formes verbales exprimant l'observation extérieure (il me sembla, il était évident...) et le doute (je n'ai jamais su, elles durent sûrement, il se peut que, etc.) (p. 132133).

Autre élément qui confirme le rôle de voix rempli par le narrateur : c'est lui qui se charge de nous donner à connaître une part des souvenirs des enfants. A plusieurs reprises, il nous raconte en effet l'épisode des pochettes surprises que sa mère - la grand-mère des filles - apportait en cadeaux à celles-ci à chaque fois qu'elle les voyait. L'enthousiasme de Mario à rapporter en détails les points insignifiants de ces petits présents donne même la sensation d'entendre directement les enfants s'extasier.

Mais outre la dissimulation possible de la part de l'enfant luimême, outre le regard externe et forcément partiel du narrateur, il y a tout simplement une limite bien humaine à tout regard :

«Soudain je les vis. Parce qu'auparavant, tant que Claudia vivait avec nous, je ne les voyais pas du tout. Elles étaient autour de nous, elles existaient, créaient des problèmes dont Claudia me parlait, elles me serraient dans leurs bras et m'embrassaient et moi aussi je les serrais dans mes bras et les embrassais, mais je ne pensais pas à elles. (...) Mais le moment était arrivé, je reconnaissais enfin les mérites de Claudia, et je compris que seulement maintenant mes filles commençaient à être véritablement les miennes. $»^{5}$.

\footnotetext{
4 Id., p. 134.

${ }^{5}$ Id., pp. 189-190.
} 
En effet, ce regard du narrateur est d'une extrême importance car celui-ci est quasiment le seul dépositaire de la voix des deux filles ; or, comment dire ce qu'on ne voit pas soi-même ? L'élément déclenchant de ce regard vrai correspond de manière non fortuite à un trait tout à fait révélateur de l'enfance : la visite chez le médecin. Par le besoin d'attention qu'il exige de ses parents, l'enfant parle :

«Peut-être les visites médicales furent-elles le premier des pas que mes filles, sans le savoir, firent pour entrer en moi, et ce pas fut suivi de beaucoup d'autres ${ }^{6}$.

Ce thème du médecin et de l'enfant maladif est d'ailleurs récurrent dans les romans de Soledad Puértolas. On le retrouve par exemple chez le personnage principal de Queda la noche, Aurora, ou encore chez celui de Una vida inesperada, etc. Dans les deux cas, la maladie permet à l'enfant de trouver sa place dans la famille $\left(1^{\mathrm{er}} \mathrm{cas}\right)$ ou dans le groupe, à l'école ( 2 ème cas) et ainsi de faire entendre sa voix. C'est le cas aussi pour Guillermo dans Días del Arenal; la faiblesse de l'enfant est lien et entrave pour la mère (p. 155). C'est pourquoi nous disons que la maladie chez l'enfant est voix.

\section{Les souvenirs d'enfance, une voix du passé pour dire le présent :}

Le troisième point de cette étude constitue ce qui fait véritablement la particularité des personnages d'enfants de Soledad Puértolas. Dans la plupart des cas, l'enfant apparaît à travers la mémoire et la voix d'un narrateur adulte, souvent l'adulte qu'il est devenu

Le souvenir est nettement signalé par l'usage de temps du passé, d'expressions telles que «je me souviens", "quand...".

Dans El bandido doblemente armado, le narrateur sans nom commence son récit au moment où, à 12 ans, il rend sa première visite à son ami Terry Lennox. C'est à partir de ces expériences d'enfance que se crée la relation du narrateur avec chacun des membres de la famille Lennox. Suite à cela, la

${ }^{6}$ Id., p. 190. 
vie du jeune homme restera irrémédiablement liée à celle des Lennox, avec des amitiés, des amours, des absences et des incompréhensions aussi. C'est ce qui constitue «l'histoire » du roman. L'enfance est assez longuement évoquée, poursuivie par une adolescence pleinement vécue :

« Je sentis que l'été n'était pas uniquement une saison. C'était un âge, notre âge. »?

Et la fin de l'enfance constitue la première rupture dans l'amitié et la relation avec Terry.

Dans le cas de El bandido doblemente armado, l'enfance est racontée de manière à la fois linéaire et partielle. Elle jette les bases de la personnalité de la vie d'adulte des personnages, mais aussi sème les jalons de l'intrigue. La description de l'enfance, puis de l'adolescence, n'est pas continue. Elle s'attache à des moments fugitifs, à des états d'âme souvent à peine ébauchés ou avoués mais bien ancrés dans l'inconscient.

Plus cruciaux encore, les souvenirs d'enfance de Guillermo dans Días del Arenal. Dans le chapitre trois, il a quarante ans et se retrouve à Madrid pour les obsèques de son père. Le texte est à la troisième personne et pourtant le narrateur emprunte tour à tour le regard du personnage et le regard de l'extérieur. Il se comporte à la fois comme un narrateur autodiégétique et extradiégétique. Ce narrateur adulte raconte des moments d'adolescence. Il retrouve sa chambre et elle le renvoie à ses 16 ans, lorsqu'une nuit entière, son père ne rentre pas à la maison. Guillermo se souvient de sa mère et de son angoisse, mais aussi du fait que pour la première fois elle avait accepté de la partager avec lui :

«Il s'enfonça dans le sommeil, comme les enfants s'endorment au milieu des tempêtes, bercés par elles. Bien qu'il ne soit plus un enfant, et que cette tempête ne soit pas non plus une tempête, un phénomène violent

${ }^{7}$ El bandido doblemente armado, p. 36. 
mais passager. Cette nuit-là avait ouvert une nouvelle époque pour les habitants de la maison. $»^{8}$.

Ce même narrateur qui semble être en mesure de retranscrire ce que le jeune Guillermo a effectivement pensé, fait un bilan de cette situation qui explique ce qu'il est à présent, en particulier vis-à-vis de son père :

«Quand arriva le moment de la jeunesse où Guillermo avait pleinement droit à son rôle de rebelle, son père avait abandonné sa mère, lui volant la vedette. Guillermo dut jouer le rôle de spectateur. Il n'avait pas pu se laisser aller à ses émotions. »9.

Ce vol de l'enfance, il ne le pardonnera jamais à son père, alors qu'il restera toujours particulièrement lié à sa mère :

« La visite chez sa mère avait pour Guillermo le goût de l'enfance, de l'ancien, de la vie connue, répétée et éternelle, de la nostalgie de tous les paradis perdus. ${ }^{10}$.

Pourtant, la remontée dans l'enfance se poursuit, le soir même des obsèques. C'est la mère qui la provoque, en expliquant comment son enfant alors âgé de 5 ans l'a empêchée, par le poids du remords, d'envisager de poursuivre une aventure avec un autre homme, et de quitter ce mari qui plus tard l'abandonnera. Ce rappel déclenche alors une véritable introspection de la part de Guillermo. Sa voix est intérieure. Mais voyons brièvement l'anecdote : en vacances à Alicante, sa mère le laisse seul l'espace d'une nuit alors qu'il dort. En effet, un homme avec lequel elle pourrait refaire sa vie lui propose une sortie et elle ne peut pas résister à la tentation. Or l'enfant se réveille et vit l'enfer de se penser abandonné et trahi. Ce traumatisme le poursuivra toute sa vie et engendrera chez lui une insécurité maladive. La mère raconte son point de vue, ses raisons, ses remords. Puis, la voix de l'enfant, longtemps refoulée, trouve enfin le droit de refaire surface :

« La longue nuit d'été à la plage dont sa mère venait de parler se reproduisit dans sa tête alors qu'il traversait les rues solitaires sans but précis. De toute sa vie, il n'avait

\footnotetext{
8 Ibid., p. 130.

9 Id., p. 145.

10 Id., p. 145.
} 
jamais pu l'oublier. Peut-être n'avait-il jamais vécu que pour cela, pour pouvoir l'oublier. (...) Qui aurait-il été s'il n'avait pas vécu cette nuit-là ? " ${ }^{11}$.

Ce n'est que lorsque l'adulte qu'il est devenu accepte d'entendre cette voix et de la faire sienne que l'enfant du passé peut se l'approprier et la revendiquer. Lorsque Guillermo revit cette scène, pour la première fois dans un roman entièrement écrit au passé, la narration se fait au présent («c'est l'été... », p. 159). Dans ce récit second, on croit entendre les questions lancinantes que se pose le petit garçon: que se passe-t-il ?, il est seul dans une maison inconnue, quelque chose de grave a dû se produire, peut-être sa mère est-elle morte, peut-être est-il seul pour toujours, peutêtre est-ce la fin du monde ? On passe avec lui par différents états d'esprit : la constatation, la solitude, le doute ; puis avec lui on prend un regard extérieur : il vit un épisode extraordinaire, c'est un héros. La terreur cède le pas quelques instants à un étrange plaisir, puis à nouveau terreur et fatigue, et enfin endormissement devant la porte de l'appartement. A son réveil, sa mère est là et lui demande pardon :

«Mais cela ne peut compenser la peur et la solitude de la nuit. Elle ignore tout de ce qu'il a pensé et souffert. Guillermo sait qu'il ne l'oubliera jamais et qu'il ne pourra parler de cela avec personne. (...) cependant, il avait toujours su que rien ne pourrait lui arracher ce souvenir de la tête, et que la douleur et l'abandon ressentis lors de cette lointaine nuit de son enfance étaient l'avant-goût de toutes les douleurs et de tous les abandons. Il fallait vivre avec ce secret, avec cette peur cachée, avec cette étrange et fragile, et à la fois insupportablement inutile, sensation d'héroïsme. " 12 .

Le passé a envahi le présent de Guillermo et de la narration. Puis le récit anecdotique reprend son cours. L'adulte Guillermo sait pourquoi il est une personne insatisfaite et indécise et il comprend sa peur de la solitude. Sa voix n'a été entendue que de lui. Mais, enfin, il s'est parlé.

${ }^{11} I d .$, p. 158.
${ }^{12}$ Id., p. 161. 
En permanence, dans plusieurs romans, le processus de remémorisation tel qu'on l'a vu ci-dessus fait naître le texte et sous-tend le cycle de l'intrigue.

Dans Todos mienten, le narrateur, Javier Arroyo, fait revivre son passé et celui de son frère Federico. Il a de son enfance, qu'il raconte au passé, le souvenir d'une mère entourée d'amies, d'allées et venues et de bruits, et les souvenirs ponctuels tournant autour des différents membres de sa famille. Souvent convoqué dans le récit d'enfance, le thème de la famille est omniprésent dans l'écriture de Soledad Puértolas.

Dans La Señora Berg, c'est encore le narrateur qui évoque son histoire personnelle. L'intrigue est soit déclenchée, soit étayée par ces recours à la mémoire. Les jalons en sont les différentes rencontres avec la voisine, mère d'un de ses camarades, la Sra Berg. Dès la première ligne du roman, le narrateur $\mathrm{y}$ fait référence et il se souvient de leurs brèves conversations puis de son attirance, et de son amour platonique. Plus tard, lorsque Mario devenu adulte revoit Marta, c'est à tour de rôle qu'ils rappellent cette adolescence par des jeux de : «Tu te souviens? ».

La voix de l'enfant remonte donc pour se répercuter et expliquer le présent de l'adulte. Le roman entier n'est qu'une suite de rencontres entre Mario et Marta, reproduisant en les développant les entrevues de l'adolescence par le rappel d'un détail ou d'une situation. L'adolescence de Mario s'épanouit à l'âge adulte.

Liés au thème de l'enfance et voix de celle-ci par delà les années, des motifs récurrents apparaissent de manière appuyée. Ces récurrences fonctionnent soit avec de simples redites, soit au travers de résumés et permettent de maintenir le lecteur dans la trame narrative. La permanence d'une idée ou d'une anecdote le rattache à un présent que l'on définit comme tel par opposition avec un passé connu.

Dans La Señora Berg, un épisode de l'enfance de Mario et de sa sœur Teresa réapparaît à plusieurs reprises : quand la famille se rendait à Novelda, chez de vieilles tantes pour le 
goûter, les deux enfants avaient le droit, pendant que les parents discutaient, de jouer sur le tapis avec un mystérieux et compliqué coffret chinois. Les deux enfants se disputaient le coffret et Teresa tentait systématiquement de prendre le dessus sur son frère. Ce cérémonial situationnel des jeux des enfants exprime leur position dans la famille ; c'est aussi une partie de leur voix. Ces jeux symbolisent les rivalités et les jalousies entre frères et sœurs. Le lien entre le passé et le présent est matérialisé par une longue phrase de onze lignes p. 105 :

« Depuis l'époque où Teresa jouait avec le coffret chinois de nos tantes de Novelda, et demeurait assise sur le tapis pendant de longues heures, se disputant silencieusement avec moi, imposant les règles du jeu, (...) chaque pas qu'elle avait accompli représentait une lente séparation par rapport à nous tous. $»^{13}$.

Le passé des deux enfants dit très exactement leur présent. Les liens entre eux sont plus que distendus. Tout les sépare. Même leurs souvenirs d'enfance ne les réunissent pas, un ressentiment croissant ayant vu le jour ; car lui, le garçon, pourtant supposé être plus solide, l'aîné, qui plus est, était maladif (encore!), délicat, sensible et rêveur et elle, au contraire, décidée, responsable.

Ces reprises régulières agissent tant au niveau de l'action que du dialogue. Et ces motifs répétés se comportent comme des fils directeurs de la lecture romanesque.

A l'identique, on retrouve les souvenirs d'infirmerie de la narratrice dans Una vida inesperada. C'est là, qu'enfant de dix ans, fragile elle aussi, elle eut le privilège de passer un après-midi entier avec Olga, légèrement plus âgée qu'elle (quinze ans), et tellement admirée de tous. Cet épisode déclenchera leur amitié, puis l'étrange relation qu'elles entretiendront toute leur vie, partagée entre l'admiration, l'identification et le rejet. Longuement décrit par la narratrice à la première personne dès la quatrième page du roman, ce moment est souvent rappelé, comme pour confirmer de

${ }^{13}$ La Señora Berg, Ed. Anagrama, 1999, p. 105. 
manière tangible dans le présent des deux femmes la réalité de ce passé fondateur.

En fait, les personnages de Soledad Puértolas donnent une impression d'unité et de cohérence qui, comme le dit Christian Boix ${ }^{14}$, " est la résultante d'une structure interne paradoxalement dialogique et polyphonique ». Les personnages s'influencent réciproquement et se connaissent les uns grâce aux autres par leurs dialogues ou leurs souvenirs. Pour dire le présent d'un adulte, une des voix qui s'élève est celle de l'enfant qu'il a été. Deux perspectives cohabitent alors, celle de l'enfant et celle de l'adulte, car la mémoire ne nous restitue le passé qu'à travers la médiation d'un regard qui, lui, appartient au présent et les commentaires du narrateur quant aux implications de la scène du coffret chinois dans $L a$ Señora Berg, en sont la preuve.

Mais, il y a forcément errance dans le souvenir. Il surgit par association d'idées ou impose une obsession, comme un leitmotiv. Au bout du compte, ces bribes de récit sur l'enfance constituent une sorte de mécanisme pour comprendre le présent à partir du passé, dans des situations actuelles ou encore dans la constitution de l'individu adulte.

Les sujets pouvant apparaître sont ceux qui sont habituels aux récits d'enfance : la famille, omniprésente chez Soledad Puértolas, avec mère et père, sœurs et frères, grands-parents, oncles, tantes et cousins, ainsi que les jeux, l'école et les amis.

Parfois, certaines scènes récurrentes, aussi anodines qu'elles puissent paraître, s'imposent comme l'objet principal de la remémoration et deviennent le centre obsessionnel du récit.

Les analepses décrivant les expériences d'un personnage perturbent la dimension chronologique du récit, accentuent les décalages entre la succession des faits de l'histoire et

14 Christian BOIX, «La voix des personnages - Pepe Carvalho dans Los Mares del Sur de Vásquez Montalbán ", actes du colloque international sur le Personnage Romanesque, Université de NiceSophia Antipolis, Avril 1994, p. 155. 
l'ordre de ces faits dans le récit. On reconnaîtra des bonds, des chevauchements et des réitérations. C'est la fonction même de la mémoire qui ne se souvient que de quelques situations isolées, souvent sans date (dans Todos mienten, le narrateur se souvient des voisins de ses grands-parents), ou avec une datation relative (par rapport à un individu, ou, souvent, à une saison). Celle-ci permet en effet une relance du récit et un ancrage dans le réel.

Le problème de la mémoire joue donc un rôle excessivement important chez Soledad Puértolas. Par son intermédiaire, le lecteur a la sensation de pénétrer dans la conscience d'un personnage. Mais, et c'est là aussi que la voix du passé sert à dire le présent, le processus de mémorisation fait appel à la faculté du lecteur d'associer les données essentielles à l'actualisation du récit et de ceux qui le composent.

L'enfant, par la référence constante qui y est faite de la part de l'adulte, propose donc ses voix multiples qui concourent à établir des liens entre les différents âges d'un même personnage, entre différents personnages, mais aussi entre ces personnages et le lecteur. Au-delà de la voix de l'enfant, nous pourrons donc parler pour ces romans de Soledad Puértolas, d'une véritable voix de l'enfance. 